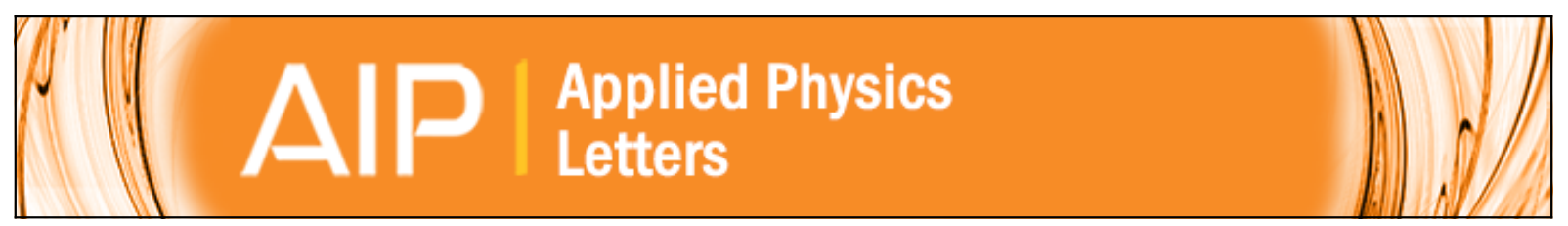

\title{
Structure of epitaxial L10-FePt/MgO perpendicular magnetic tunnel junctions
}

Amit Kohn, Nadav Tal, Ayala Elkayam, Andras Kovàcs, Dalai Li, Shouguo Wang, Saman Ghannadzadeh,

Thorsten Hesjedal, and Roger C. C. Ward

Citation: Applied Physics Letters 102, 062403 (2013); doi: 10.1063/1.4791576

View online: http://dx.doi.org/10.1063/1.4791576

View Table of Contents: http://scitation.aip.org/content/aip/journal/apl/102/6?ver=pdfcov

Published by the AIP Publishing

\section{Articles you may be interested in}

Effect of interfacial structures on spin dependent tunneling in epitaxial L10-FePt/MgO/FePt perpendicular magnetic tunnel junctions

J. Appl. Phys. 117, 083904 (2015); 10.1063/1.4913265

Correlation of the structural properties of a Pt seed layer with the perpendicular magnetic anisotropy features of full Heusler-based Co2FeAl/MgO/Co2Fe6B2 junctions via a 12-inch scale Si wafer process

Appl. Phys. Lett. 103, 162409 (2013); 10.1063/1.4824306

Effects of Pt capping layer on perpendicular magnet anisotropy in pseudo-spin valves of

$\mathrm{Ta} / \mathrm{CoFeB} / \mathrm{MgO} / \mathrm{CoFeB} / \mathrm{Pt}$ magnetic-tunneling junctions

Appl. Phys. Lett. 102, 212409 (2013); 10.1063/1.4808084

Perpendicular-anisotropy CoFeB-MgO magnetic tunnel junctions with a $\mathrm{MgO} / \mathrm{CoFeB} / \mathrm{Ta} / \mathrm{CoFeB} / \mathrm{MgO}$ recording structure

Appl. Phys. Lett. 101, 022414 (2012); 10.1063/1.4736727

Modeling of the spin-transfer torque switching in FePt/MgO-based perpendicular magnetic tunnel junctions: A combined ab initio and micromagnetic simulation study

Appl. Phys. Lett. 99, 032508 (2011); 10.1063/1.3615664

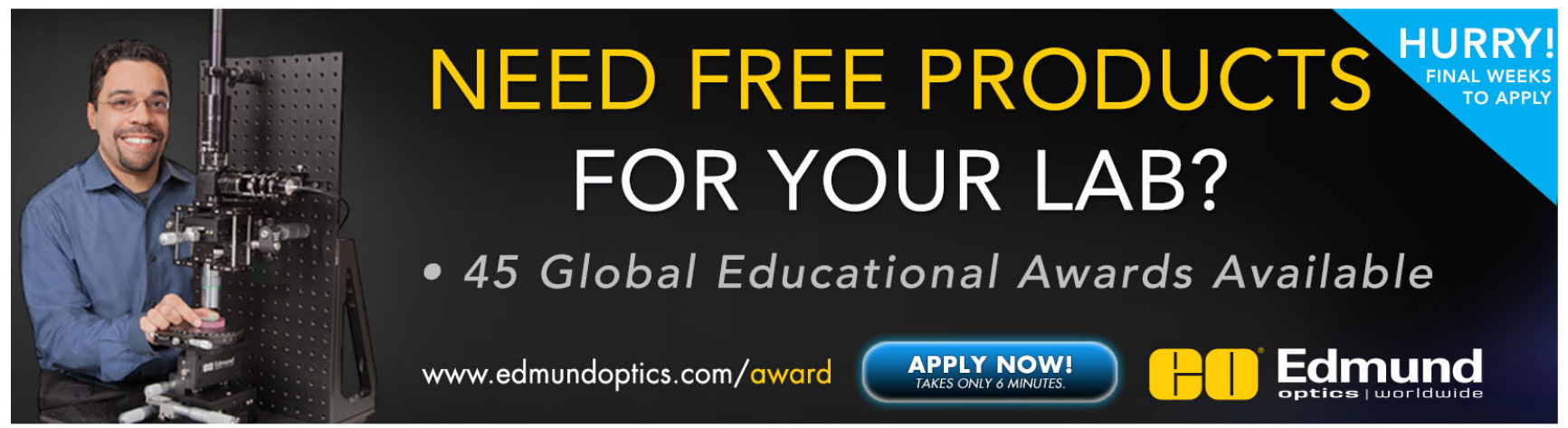




\title{
Structure of epitaxial $\mathrm{L}_{1}-\mathrm{FePt} / \mathrm{MgO}$ perpendicular magnetic tunnel junctions
}

\author{
Amit Kohn, ${ }^{1, a)}$ Nadav Tal, ${ }^{1}$ Ayala Elkayam, ${ }^{1}$ Andras Kovàcs, ${ }^{2}$ Dalai Li, ${ }^{3}$ Shouguo Wang, ${ }^{3, a)}$ \\ Saman Ghannadzadeh, ${ }^{4}$ Thorsten Hesjedal, ${ }^{4}$ and Roger C. C. Ward ${ }^{4, a)}$ \\ ${ }^{1}$ Department of Materials Engineering, Ilse Katz Institute for Nanoscale Science and Technology, \\ Ben-Gurion University of the Negev, Beer-Sheva 84105, Israel \\ ${ }^{2}$ Ernst Ruska-Centre for Microscopy and Spectroscopy with Electrons, Peter Grünberg Institute, \\ Forschungszentrum Jülich, Jülich 52425, Germany \\ ${ }^{3}$ State Key Laboratory of Magnetism, Beijing National Laboratory for Condensed Matter Physics, \\ Institute of Physics, Chinese Academy of Sciences, Beijing 100190, China \\ ${ }^{4}$ Clarendon Laboratory, Department of Physics, University of Oxford, Oxford OX1 3PU, United Kingdom
}

(Received 12 November 2012; accepted 28 January 2013; published online 11 February 2013)

\begin{abstract}
Perpendicular magnetic tunnel junctions (p-MTJs) with $\mathrm{MgO}$ barriers are interesting for high-density information-storage devices. Chemically ordered $\mathrm{L}_{0}-\mathrm{FePt}$ is a potential electrode due to its large perpendicular magnetocrystalline anisotropy. To-date, a single theoretical study on $\mathrm{L1}_{0}-\mathrm{FePt} / \mathrm{MgO} \mathrm{p}$-MTJ based on an idealized structure reported significant dependence of spin-dependent tunneling on interface structure. [Y. Taniguchi et al., IEEE Trans. Magn. 44, 2585 (2008).] We report a structural study of epitaxial $\mathrm{L}_{0_{0}}-\mathrm{FePt}(001)[110] / / \mathrm{MgO}(001)[110] / / \mathrm{L} 1_{0^{-}}$ $\mathrm{FePt}(001)[110] \mathrm{p}$-MTJs, focusing on the interfaces using aberration-corrected scanning transmission electron microscopy. Interfaces are semi-coherent, with oxygen atomic-columns of $\mathrm{MgO}$ located opposite to iron atomic-columns in $\mathrm{L}_{0}-\mathrm{FePt}$. Up to three lattice planes show atomiccolumn steps, the origin of which is attributed to antiphase boundaries in $\mathrm{L1}_{0}$-FePt. (C) 2013 American Institute of Physics. [http://dx.doi.org/10.1063/1.4791576]
\end{abstract}

MTJs are used as read-head sensors for hard-diskdrives and memory units in magnetoresistive random access memory. For magnetic recording, increasing the storage density is limited by superparamagnetism, which is a fundamental limitation for future development. ${ }^{1}$ p-MTJs based on electrodes with large perpendicular magnetic anisotropy are promising for higher information-storage densities and thermal stability. ${ }^{2}$ Furthermore, p-MTJs may reduce critical current densities for magnetization reversal by spin-transfer torque, while maintaining thermal stability. ${ }^{3,4}$ One such electrode material is chemically ordered $\mathrm{L}_{0}-\mathrm{FePt}$ alloy (body-centered-tetragonal, $\mathrm{CuAu}$ phase prototype, spacegroup C4/mmm, $a=b=0.3849 \mathrm{~nm}$ and $c=0.3700 \mathrm{~nm}$ (Ref. 5)), in which the perpendicular magnetocrystalline anisotropy (c-axis) constant, $K_{u}$, is $\sim 10^{7} \mathrm{erg} / \mathrm{cm}^{3} .{ }^{6-9}$ MTJs using crystalline $\mathrm{MgO}$ tunnel barriers and electrodes with in-plane magnetization have been studied intensively due to spin-filtering of the barrier resulting in large tunneling magneto-resistance (TMR) ratios. ${ }^{10-12} \mathrm{FePt} / \mathrm{MgO}$ MTJs have also been shown experimentally to exhibit high TMR values. ${ }^{13}$ Previous reports on $\mathrm{L}_{0}$-FePt electrodes dealt with the dependence of TMR ratio on the degree of chemical-ordering, ${ }^{14}$ electrode thickness, ${ }^{15}$ and epitaxial growth on $\mathrm{Au}(001)$ surfaces. ${ }^{16}$

To date, a single theoretical study has examined the effect of the $\mathrm{L}_{0}$-FePt p-MTJ structure, in particular the $\mathrm{FePt} / \mathrm{MgO}$ interface, on spin-dependent tunnelling. ${ }^{17}$ Taniguchi and co-workers predict that Fe-terminated interfaces result in higher TMR ratios (380\%) than Pt-terminated inter-

\footnotetext{
a) Authors to whom correspondence should be addressed. Electronic addresses: akohn@exchange.bgu.ac.il,sgwang@iphy.ac.cn, and r.ward2@ physics.ox.ac.uk.
}

faces $(70 \%)$. Additionally, they report that Fe-terminated interfaces are energetically favorable, and that iron atoms are located opposite to oxygen atoms. The p-MTJ structure assumed for this first-principles calculation was ideal, namely: coherent, atomically flat interfaces and lack of structural defects, despite $\sim 9 \%$ lattice mismatch between $\mathrm{L}_{0^{-}}$ $\mathrm{FePt}$ and $\mathrm{MgO}$. However, structural characterization, at atomic-scale spatial resolution of $\mathrm{L} 1_{0}$-FePt based p-MTJs, is lacking, in particular regarding the structure of the interface between the electrode and the tunneling barrier. This information is necessary for theoretical calculations leading to an understanding of the spin-dependent tunneling mechanism, and hence possibly increasing TMR ratios. Therefore, we report an atomic-scale structural characterization, focusing on the interface structure between electrode and barrier, of $\mathrm{L}_{0}$-FePt/MgO p-MTJs prepared by molecular-beam-epitaxy (MBE) growth. Though sputter-deposition is well-suited for technological applications and demonstrated high TMR ratios (e.g., Ref. 13), MBE-grown epitaxial MTJs can achieve high crystal quality, well-defined interface structures, and therefore be studied as model systems.

Epitaxial $\mathrm{FePt}(5 \mathrm{~nm}) / \mathrm{MgO}(3 \mathrm{~nm}) / \mathrm{FePt}(20 \mathrm{~nm}) \quad$ MTJs were MBE-grown (base pressure $\sim 5 \times 10^{-11}$ mbar) on single-crystal $\mathrm{MgO}(001)$ substrates. Because of the $9 \%$ lattice mismatch between $\mathrm{MgO}$ and $\mathrm{FePt}(001), \mathrm{Cr}$ buffer and $\mathrm{Pt}$ seed layers were used to improve crystalline perfection of the bottom FePt layer, as suggested by Gehanno et al. ${ }^{18} \mathrm{FePt}$ layers were grown by co-evaporation of high-purity $\mathrm{Fe}$ and Pt with controlled deposition rates to achieve the stoichiometric alloy. The $\mathrm{MgO}$ barrier was grown by e-beam evaporation of a source material of the same purity as the $\mathrm{MgO}$ substrate. Samples were capped with $\mathrm{Cr}$ for protection. During deposition, the structure of each layer was monitored in- 
situ by reflection high-angle electron diffraction. Subsequent measurements with spherical-aberration, Cs, corrected bright-field STEM show the number of $\mathrm{MgO}(002)$ crystallographic planes in the barrier between 10 and 12 , demonstrating the accuracy of MBE-growth in fabricating p-MTJs. At a substrate temperature of $500{ }^{\circ} \mathrm{C}$, FePt grew directly in the ordered $\mathrm{L1}_{0}$-phase with [001] axis normal to the substrate plane. Full ordering, therefore, corresponds to sequencing of alternate $\mathrm{Fe}$ and $\mathrm{Pt}(001)$ planes along the growth direction.

The degree of order achieved was measured by growing thicker FePt layers for X-ray diffraction (XRD, PANalytical X'Pert) analysis. Figure 1 shows a longitudinal-XRD scan from a single $25 \mathrm{~nm}$ thick layer grown under identical conditions as the junctions. Additional (001) and (003) superlattice reflections due to the ordered FePt structure are clearly visible. Figure 1 (inset) shows the $\mathrm{FePt}(002)$ reflection from a $\mathrm{FePt}(25 \mathrm{~nm}) / \mathrm{MgO}(3 \mathrm{~nm}) / \mathrm{FePt}(50 \mathrm{~nm})$ sample. The doublet indicates that the top FePt layer is under higher tensile strain than the bottom layer. From the longitudinal scans, we measured $c=0.370 \mathrm{~nm}$ and $c=0.367 \mathrm{~nm}$ for the bottom and top layers, respectively (all $\pm 0.001 \mathrm{~nm}$ ). The relative areas of superlattice and fundamental peaks are used to calculate the chemical-ordering parameter, $S$, where $S=0$ and $S=1$ correspond to a random alloy and ideal ordered structure, respectively. ${ }^{19}$ By comparing integrated intensities of (001) and (002) reflections recorded from the $\mathrm{FePt}(25 \mathrm{~nm}) / \mathrm{MgO}(3 \mathrm{~nm}) /$ $\mathrm{FePt}(50 \mathrm{~nm})$ sample, we calculate $S=0.92$ and $S=0.88$ for bottom and top layers, respectively. These calculations account for Lorentz polarisation, Debye-Waller, and absorption factors. A high-degree of $\mathrm{L}_{0}$ order has, therefore, been achieved in MBE-grown FePt layers.

TMR ratios were measured (reported separately in Ref. 20) by patterning multilayer stacks into elliptical junctions $\left(4 \times 8 \mu \mathrm{m}^{2}\right)$ using ultra-violet optical-lithography and $\mathrm{Ar}$ ion-beam etching. The p-MTJs were measured by a physicalproperties-measurement-system using a standard four-probe technique measuring TMR ratios of $38 \%$ and $16 \%$ at $5 \mathrm{~K}$ and room-temperature, respectively. Detailed information regarding growth and MTJ fabrication was reported previously. .1,22 $^{21}$

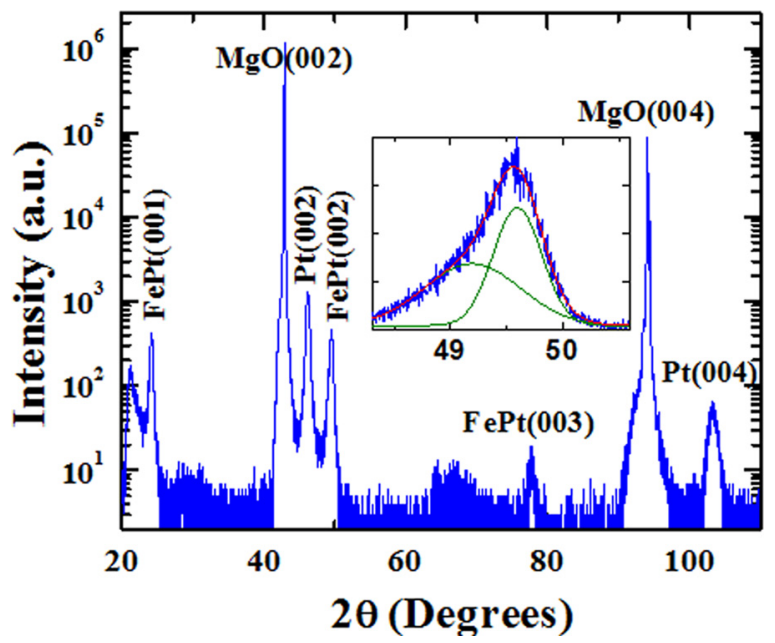

FIG. 1. Longitudinal-XRD scan from a $25 \mathrm{~nm}$ thick FePt layer grown under identical conditions as p-MTJs. (inset) $\mathrm{FePt}(002)$ reflection from a $\mathrm{FePt}(25 \mathrm{~nm}) / \mathrm{MgO}(3 \mathrm{~nm}) / \mathrm{FePt}(50 \mathrm{~nm})$ sample, in which the doublet indicates that the top FePt layer is under higher tensile strain than the bottom layer.
The overall crystallographic structure of the MTJs was determined by conventional high-resolution transmission electron microscopy (phase-contrast, HR-TEM), and selected area electron diffraction (SAED), using a JEOL JEM-2100F. Cscorrected high-angle annular dark-field (HAADF) STEM was chosen to characterize the structure of the $\mathrm{L}_{0}-\mathrm{FePt} / \mathrm{MgO}$ interface. An FEI-Titan was operated at $300 \mathrm{kV}$ accelerating voltage, convergence semi-angle of $25 \mathrm{mrad}$, and beam diameter of $0.1 \mathrm{~nm}$, thus achieving chemically sensitive atomicscale contrast without delocalization of information. Chemical intermixing at the interface was assessed by parallel acquisition of HAADF-STEM and electron energy-loss spectroscopy (EELS) signals along atomic planes perpendicular to the growth direction, using the "StripeSTEM" methodology. ${ }^{23}$ EEL spectra and HAADF data were recorded simultaneously such that each spectrum is related to several scan lines in the HAADF image, therefore corresponding to a lattice plane. EEL spectra were recorded using a Gatan imaging filter (GIF) Tridiem, at a collection semi-angle of $18 \mathrm{mrad}$. Crosssectional TEM samples were prepared by tripod mechanical polishing and low-energy $(<1 \mathrm{keV})$ ion-milling.

The proposed interface structure was tested by comparing experimental HAADF-STEM images to multislice image simulations. ${ }^{24}$ The starting model for image simulations was the ideal interface proposed by Taniguchi et al., ${ }^{17}$ namely coherent interfaces in which the $\mathrm{L}_{0}$-FePt electrode is terminated with an $\mathrm{Fe}$ layer, $\mathrm{Fe}$ atoms located opposite to $\mathrm{O}$ atoms at a distance of $0.22 \mathrm{~nm}, \mathrm{MgO}$ strained in-plane to the bulk FePt lattice, and fully relaxed interlayer separations. For image simulations, a frozen-phonon model was applied, with inner and outer HAADF detector angles of 78 and $400 \mathrm{mrad}$, respectively.

An overview of the MTJ multilayer is shown in Fig. 2, which is a conventional HRTEM cross-sectional image obtained along the $\mathrm{MgO}[1-10]$ zone-axis. Power-spectra (optical diffractograms) from the layers, as well as SAED (not shown here) from the entire structure verify the epitaxial relations: $\mathrm{Pt}(001)[110] / / \mathrm{L1}_{0}-\mathrm{FePt}(001)[110] / / \mathrm{MgO}(001)[110] / /$ $\mathrm{L1}_{0}-\mathrm{FePt}(001)[110]$.

The interface between the bottom electrode and barrier is semi-coherent as demonstrated in Fig. 2(e). A powerspectrum from a region including both bottom electrode and barrier shows splitting of the (220) reflection indicating inplane relaxation of the $\mathrm{MgO}$ barrier. The reconstructed image (inset, center) using this (220) reflection shows mostly regions of a coherent interface, as well as dislocations, for example at positions marked schematically by the red arrow. The misfit dislocations seen here are spaced approximately 14 atomic planes along the $\mathrm{FePt} / \mathrm{MgO}$ interface, which is consistent with a relaxation of a $7 \%$ lattice mismatch.

Figure 3(a) is a Cs-corrected HAADF-STEM crosssectional image of the $\mathrm{FePt} / \mathrm{MgO}$ p-MTJ. In these images, $\mathrm{Fe}$ and $\mathrm{Pt}$ atomic-columns are separated clearly due to large differences in atomic numbers $\left(Z_{F e}=26\right.$ and $\left.Z_{P \mathrm{t}}=78\right)$ of constituent atoms, i.e., Pt atomic-columns appear brighter than Fe atomic-columns. When examining the interface between the Pt seed-layer and FePt electrode, atomic-column steps are observed, which we then attribute as the cause for antiphase boundaries in the electrode (described later). The location of the $\mathrm{Pt} / \mathrm{FePt}$ interface was determined by inspecting 

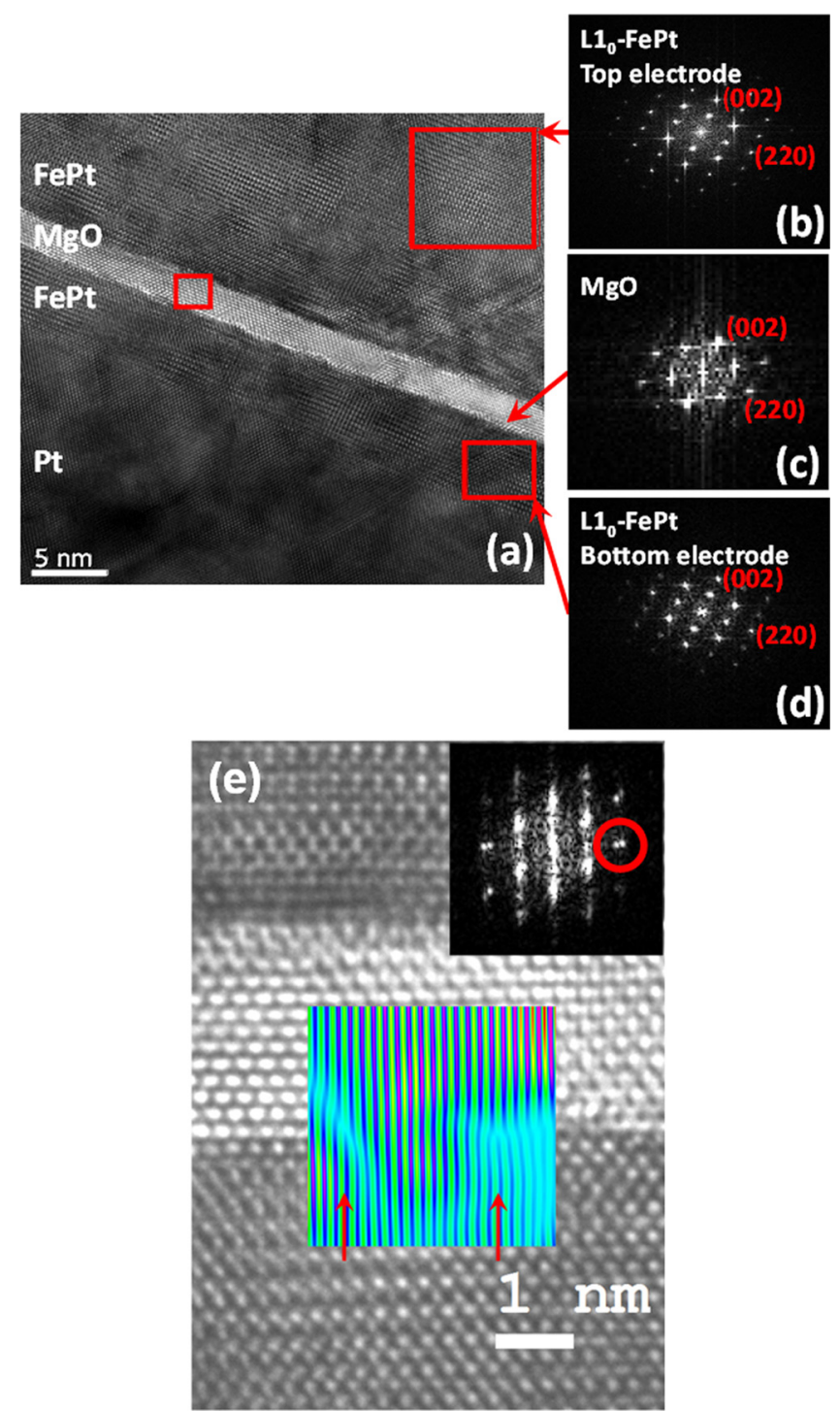

FIG. 2. (a) Cross-sectional HRTEM image of the entire p-MTJ structure obtained along the $\mathrm{MgO}[1-10]$ direction. Power-spectra from regions denoted schematically by the red squares in (b) top FePt electrode, (c) $\mathrm{MgO}$ barrier, and (d) bottom FePt electrode. These power spectra show the $\mathrm{L}_{0}-\mathrm{FePt}(001)[110] / / \mathrm{MgO}(001)[110] / / \mathrm{L1}_{0}-\mathrm{FePt}(001)[110]$ epitaxial relation and confirm the $\mathrm{L}_{0}$ structure of FePt electrodes. (e) HRTEM crosssectional image of the $\mathrm{FePt}$ electrodes and $\mathrm{MgO}$ barrier. Inset (top right): power-spectrum from a region including the bottom electrode and barrier shows splitting of the (220) reflection, marked schematically by the red circle. Inset (center): reconstructed image of the semi-coherent interface between the bottom $\mathrm{FePt}$ electrode and $\mathrm{MgO}$ barrier using the (220) reflection in which misfit dislocations are marked schematically by the red arrow.

image intensity variations along diagonal line-scans (FePt $\langle 110\rangle$ direction), through both the Pt seed layer and the FePt electrode (red circles shown in Fig. 3(a) mark schematically positions where intensity line scans were examined). In the electrode, HAADF image intensity peaks alternate between higher intensity for $\mathrm{Pt}$ and lower intensity for Fe, while for the Pt seed, all peaks are of the same intensity. Thus, the Pt/ FePt interface was located at the termination of intensity oscillations. Distances between atomic-column steps, marked schematically by red lines in Fig. 3(a), range between 2 and $4 \mathrm{~nm}$. In order to examine the effect of these atomic-column steps on subsequent growth of the electrode, the HAADF-STEM image was reconstructed from the

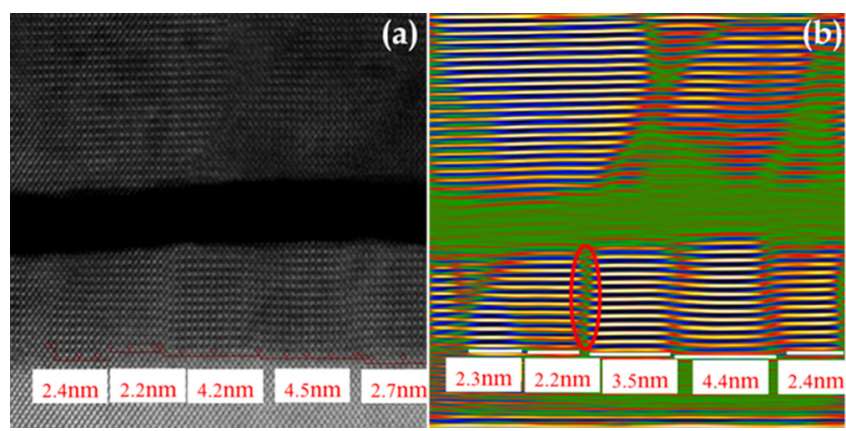

FIG. 3. (a) HAADF-STEM (inner detector semi-angle: $78 \mathrm{mrad}$ ) image demonstrating steps at the interface of the Pt seed-FePt bottom electrode (denoted schematically by red lines). (b) Image constructed from the powerspectrum of (a) using the FePt (002) reflection (perpendicular to growth direction), which highlights antiphase boundaries, as shown, for example, in the region of the red oval.

power-spectrum using the $\mathrm{FePt}(002)$ reflection (growth direction). Figure 3(b) shows such an image reconstruction, which represents a typical case out of 20 regions examined. This reconstructed image highlights regions with reduced ordering of the $\mathrm{FePt}(002)$ crystallographic plane, thus locating antiphase boundaries. For example, Fig. 3(b), shows such an antiphase boundary, the location of which is shown schematically by the red oval. The location and distances between antiphase boundaries, ranging between 2 and $4 \mathrm{~nm}$, agree with the positions of atomic-columns steps at the interface between FePt and Pt. Figure 3(b) also indicates that the epitaxial quality of the top FePt electrode is reduced compared to the bottom electrode. At the bottom electrode, antiphase boundaries are confined to narrow regions and are spread evenly. However, the top electrode shows larger disordered regions, which represent poorer (002) periodicity of the crystal. This observation is expected because of the greater lattice mismatch for growth of the top electrode on $\mathrm{MgO}$, compared with the bottom electrode on the Pt seed.

Atomic-column steps are observed also at the interface between the $\mathrm{L1}_{0}-\mathrm{FePt}$ electrode and the $\mathrm{MgO}$ barrier. Figure 4 is a HAADF-STEM image focusing on the interface between the bottom electrode and barrier. To reduce highfrequency noise related to instrumental instabilities, a lowpass filter (Butterworth filter, 0.5 weighting) was applied, and to reduce noise related to inelastic scattering, image contrast was improved by masking elastic reflections of $\mathrm{FePt}$ and $\mathrm{MgO}$ layers in the power-spectra and then reconstructing the image. The resulting contrast of these images differentiates clearly between $\mathrm{Pt}, \mathrm{Fe}$, and $\mathrm{Mg}$ atomic-columns though is not sensitive to oxygen atomic-columns due to its low atomic number relative to $\mathrm{Fe}$ and $\mathrm{Pt}$. At the electrode-barrier interfaces, we observe several atomic layers, which do not follow a well-defined chemical composition. This region is typically 1-2 atomic layers thick at the bottom electrode interface, and 2-3 atomic layers thick at the top electrode interface. The example presented in Fig. 4 shows two unresolved layers between the barrier and a Fe-terminated bottom electrode. Intermixing at the interface is demonstrated using HAADF intensity line scans along crystallographic planes, as presented schematically by green rectangles in Fig. 4. Line scans \#1 and \#3, located on Pt atomic-planes, show intensity differences between maxima and background signals 


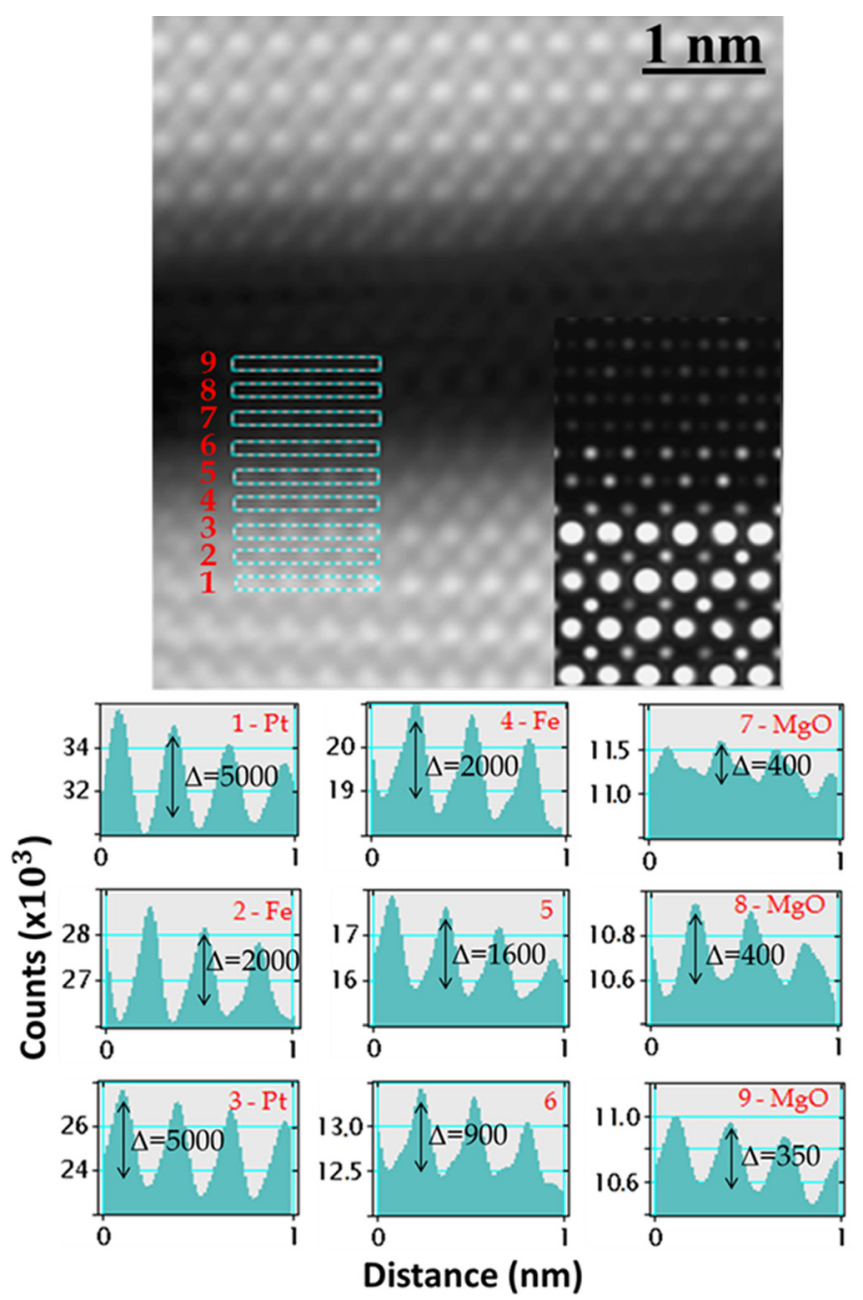

FIG. 4. (Left) Processed HAADF-STEM image of the FePt/MgO/FePt interfaces, inset: image simulation of an Fe-terminated interface and atomiccolumn steps at the interface between barrier and electrode. (Right) Intensity line scans along the $\mathrm{Pt}, \mathrm{Fe}$, and $\mathrm{MgO}$ crystallographic planes, marked schematically by the green rectangles in the HAADF-STEM image. The image intensity differences demonstrate two crystallographic planes (line scans \#5 and \#6), which cannot be attributed either to the FePt electrode or MgO barrier.

of $\sim 5000$ counts. For line scans \#2 and \#4, located on Fe atomic-planes, intensity differences are $\sim 2000$ counts. On the barrier side, line scans \#7-\#9, located on $\mathrm{Mg}-\mathrm{O}$ planes, and experimentally sensitive only to $\mathrm{Mg}$ atomic-columns, show intensity differences of $\sim 400$ counts. Between the barrier and electrode, line scans \#5 and \#6, the intensity differences of 1600 and 900 counts, respectively, are lower than for $\mathrm{Fe}$ atomic-planes yet larger than for $\mathrm{MgO}$ planes. We note that for all examined images, the termination layer of the electrodes, before these intermediate layers, is attributed to a Fe plane based on this approach. Due to the relative position of the $\mathrm{Fe}$ and $\mathrm{Mg}$ peaks in these line scans, and according to comparisons to image simulations (see following), we conclude that oxygen atomic-columns in the first layer of the barrier are located opposite to $\mathrm{Fe}$ atomiccolumns in the electrode. This latter result is consistent with the theoretical conclusion of Taniguchi et al. based on a comparison of adhesion energies of the interface. ${ }^{17}$ We propose that the intermediate layers are related to atomiccolumn steps at the interface in the direction of the electron probe similar to those observed laterally at the FePt/Pt interface. To examine this assumption, image simulations and EELS line scans were undertaken.

The proposed structure of the interface was tested by comparing experimental HAADF-STEM images to multislice image-simulations ${ }^{24}$ as shown in Fig. 4 (inset). The starting model for image simulations was the ideal interface proposed by Taniguchi et al. ${ }^{17}$ of coherent interfaces in which the electrode is terminated with an Fe layer, Fe atoms located opposite to $\mathrm{O}$ atoms at a distance of $0.22 \mathrm{~nm}, \mathrm{MgO}$ strained in-plane with respect to bulk $\mathrm{L}_{0}-\mathrm{FePt}$ lattice, and fully relaxed interlayer separations. Atomic-column steps are assumed in the two layers between a Fe terminated FePtelectrode and $\mathrm{MgO}$-barrier. This image simulation is in qualitative agreement with experimental results in terms of intensities and location of atomic columns.

The chemical composition of the interface was characterized using the "StripeSTEM" EELS method ${ }^{23}$ in order to measure the relative atomic composition of $\mathrm{Fe}$ and $\mathrm{O}$ across the $\mathrm{FePt} / \mathrm{MgO}$ interfaces. Figure 5 shows a compositional profile across the bottom $\mathrm{FePt} / \mathrm{MgO}$ interface calculated from $\mathrm{Fe}-\mathrm{L}$ and $\mathrm{O}-\mathrm{K}$ core-loss edges using hydrogenic (white-lines) cross-section model. Multivariate statistical analysis improved the signal to noise ratio of the spectra by using the first seven calculated eigenvalues. ${ }^{25,26}$ Interface chemical intermixing was estimated by fitting the compositional profile of $\mathrm{Fe}$ and $\mathrm{O}$ to an error function. The width of the interface, which is a measure of chemical intermixing, was defined as the distance between fractions of 0.16 and 0.84 of maximum $\mathrm{Fe}$ or $\mathrm{O}$ content at the onset of the interface. Applying this analysis to ten regions in the sample, the width of the bottom interface is $0.84 \pm 0.18 \mathrm{~nm}$, while the top interface is wider, $1.21 \pm 0.04 \mathrm{~nm}$. To examine whether

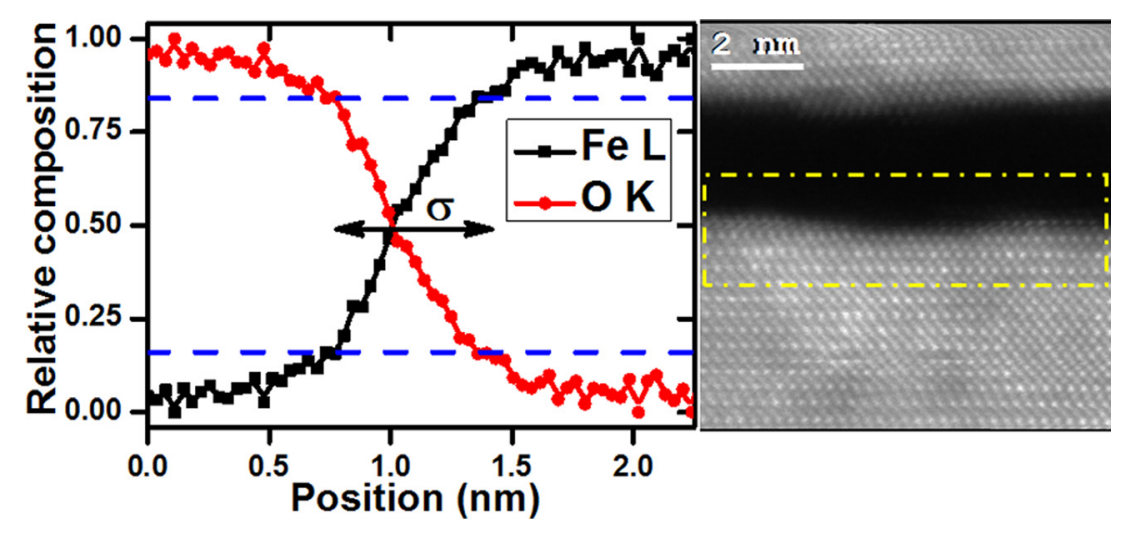

FIG. 5. (Left) Example of relative Fe and O composition across the bottom $\mathrm{FePt} / \mathrm{MgO}$ interface (from the area of the dashed yellow rectangle, right) calculated using the Fe L and $\mathrm{O} \mathrm{K}$ core-loss edges in EEL spectra when recording the HAADF-STEM image (right) using the "StripeSTEM" approach. 
this result is a measurement artifact due to broadening of the electron probe resulting from sample thickness, we applied a geometrical model described in Ref. 27, assuming all electrons were collected by the spectrometer. The sample thickness was estimated by the ratio of zero-loss to plasmon peaks, at $\sim 0.22$ units of inelastic mean free path (IMFP). The IMFP value for FePt was calculated at $89 \mathrm{~nm},{ }^{28}$ namely an estimated specimen thickness of $20 \mathrm{~nm}$, in agreement with the value obtained from comparisons of bright-field STEM images to image simulations. Consequently, geometric broadening is $\sim 0.36 \mathrm{~nm}$, and considering the beam diameter of $0.1 \mathrm{~nm}$, the overall beam broadening is under $0.4 \mathrm{~nm}$. Therefore, the widths of interfaces determined experimentally are wider than those expected for sharp interfaces with beam broadening. We estimate the compositional width of the interface at approximately $0.7 \mathrm{~nm}$, equivalent to 2-3 crystallographic planes in the FePt[001] direction. We suggest that the reason for the chemical intermixing at the interface is due to atomic steps in the direction of the electron beam and in the scanning direction, as also proposed above based on atomic-column resolution HAADF-STEM images.

In summary, we presented atomic-scale characterization of MBE-grown $\mathrm{L}_{0}$-FePt/MgO p-MTJs focusing on the structure of interfaces between electrodes and tunneling barrier. We showed that interfaces are semi-coherent with atomic steps, which give rise to intermediate layers, of up to three atomic-planes, where the chemical composition is not well defined over the thickness of the TEM samples. The terminating layer prior to this intermixing region is identified as a Fe atomic plane. Oxygen atomic-columns in the barrier are located opposite to iron atomic-columns in the $\mathrm{L} 1_{0}-\mathrm{FePt}$ electrode. We observe antiphase boundaries in the electrodes, attributed to atomic steps at the interface between the $\mathrm{Pt}$ seed layer and bottom $\mathrm{L}_{1}$-FePt electrode. A comparison of the electrodes shows that the epitaxial quality of the top electrode is reduced compared with the bottom electrode.

A.K. and S.W. acknowledge the support for this research by a Grant from the Ministry of Science \& Technology, P.R.China (China-Israel project with Grant No. 2013DFG13020) and the Ministry of Science \& Technology, Israel. The authors are grateful to Keith Belcher for technical support of the MBE growth. R.C.C.W. and T.H. acknowledge the support of the Clarendon Laboratory at Oxford University for the MBE growth of the samples. A.K. acknowledges the support of the Ilse Katz Institute for Nanoscale Science and Technology for the electron microscopy characterization. S.W. acknowledges the financial support of the National Basic Research Program of China (Grant No. 2009CB929203) and the National Natural Science Foundation of China (Grant Nos. 50972163 and 11274371) for the fabrication of the p-MTJs and the magneto-transport measurements.

${ }^{1}$ S. N. Piramanayagam, J. Appl. Phys. 102, 011301 (2007).

${ }^{2}$ J. M. Slaughter, Annu. Rev. Mater. Res. 39, 277 (2009).

${ }^{3}$ A. D. Kent, B. Özyilmaz, and E. Barco, Appl. Phys. Lett. 84, 3897 (2004).

${ }^{4}$ K. J. Lee, O. Redon, and B. Dieny, Appl. Phys. Lett. 86, 022505 (2005).

${ }^{5}$ S. Yuasa, H. Miyajima, and Y. Otani, J. Phys. Soc. Jpn. 63, 3129 (1994).

${ }^{6}$ D. Weller, A. Moser, L. Folks, M. E. Best, W. Lee, M. F. Toney, M. Schwickert, J.-U. Thiele, and M. F. Doerner, IEEE. Trans. Magn. 36, 10 (2000).

${ }^{7}$ J. Yu, U. Ruediger, A. D. Kent, R. F. C. Farrow, R. F. Marks, D. Weller, L. Folks, and S. S. P. Parkin, J. Appl. Phys. 87, 6854 (2000).

${ }^{8}$ S. Okamoto, N. Kikuchi, O. Kitakami, T. Miyazaki, Y. Shimada, and K. Fukamichi, Phys. Rev. B 66, 024413 (2002).

${ }^{9}$ P. de Person, P. Warin, M. Jamet, C. Beigne, and Y. Samson, Phys. Rev. B 76, 184402 (2007).

${ }^{10}$ S. S. P. Parkin, C. Kaiser, A. Panchula, P. M. Rice, B. Hughes, M. Samant, and S. H. Yang, Nature Mater. 3, 862 (2004).

${ }^{11}$ S. Yuasa, T. Nagahama, A. Fukushima, Y. Suzuki, and K. Ando, Nature Mater. 3, 868 (2004).

${ }^{12}$ W. H. Butler, X.-G. Zhang, and T. C. Schulthess, Phys. Rev. B 63, 054416 (2001).

${ }^{13}$ M. Yoshikawa, E. Kitagawa, T. Nagase, T. Daibou, M. Nagamine, K. Nishiyama, T. Kishi, and H. Yoda, IEEE Trans. Magnet. 44, 2573 (2008).

${ }^{14}$ T. Moriyama, S. Mitani, T. Seki, T. Shima, and K. Takanashi, J. Appl. Phys. 95, 6789 (2004).

${ }^{15}$ N. Inami, G. Kim, T. Hiratsuka, H. Naganuma, M. Oogane, and Y. Ando, J. Phys.: Conf. Ser. 200, 052008 (2010).

${ }^{16}$ M. Hagiuda, S. Mitani, T. Seki, K. Yakushiji, T. Shima, and K. Takanashi, J. Magn. Magn. Mater. 310, 1905 (2007).

${ }^{17}$ Y. Taniguchi, Y. Miura, K. Abe, and M. Shirai, IEEE Trans. Magn. 44, 2585 (2008)

${ }^{18}$ V. Gehanno, Y. Samson, A. Marty, B. Gilles, and A. Chamberod, J. Magn. Magn. Mater. 172, 26 (1997).

${ }^{19}$ B. E. Warren, X-Ray Diffraction (Dover, New York, 1990).

${ }^{20}$ Q. L. Ma, S. G. Wang, S. H. Liang, H. F. Liu, D. L. Li, T. Hesjedal, R. C. C. Ward, and X. F. Han, "Transport properties of perpendicular FePt(001)/ $\mathrm{MgO} / \mathrm{FePt}$ fully epitaxial magnetic tunnel junctions" (unpublished).

${ }^{21}$ S. G. Wang, R. C. C. Ward, G. X. Du, X. F. Han, C. Wang, and A. Kohn, Phys. Rev. B 78, R180411 (2008).

${ }^{22}$ C. Wang, A. Kohn, S. G. Wang, L. Y. Chang, S.-Y. Choi, A. I. Kirkland, A. K. Petford-Long, and R. C. C. Ward, Phys. Rev. B 82, 024428 (2010).

${ }^{23}$ M. Heidelmann, J. Barthel, and L. Houben, Ultramicroscopy 109, 1447 (2009).

${ }^{24}$ R. Kilaas, MacTempasX, Total Resolution LLC (2012).

${ }^{25} \mathrm{P}$. Trebbia and C. Mory, Ultramicroscopy 34, 179 (1990).

${ }^{26}$ S. Lozano-Perez, V. D. Bernal, and R. J. Nicholls, Ultramicroscopy 109, 1217 (2009).

${ }^{27}$ R. F. Egerton, Ultramicroscopy 107, 575 (2007).

${ }^{28}$ T. Malis, S. C. Cheng, and R. F. Egerton, J. Electron Microsc. Technol. 8, 193 (1988). 